\title{
Precise time scales and navigation systems: mutual benefits of timekeeping and positioning
}

\author{
Patrizia Tavella* and Gérard Petit
}

\begin{abstract}
The relationship and the mutual benefits of timekeeping and Global Navigation Satellite Systems (GNSS) are reviewed, showing how each field has been enriched and will continue to progress, based on the progress of the other field. The role of GNSSs in the calculation of Coordinated Universal Time (UTC), as well as the capacity of GNSSs to provide UTC time dissemination services are described, leading now to a time transfer accuracy of the order of 1-2 ns. In addition, the fundamental role of atomic clocks in the GNSS positioning is illustrated. The paper presents a review of the current use of GNSS in the international timekeeping system, as well as illustrating the role of GNSS in disseminating time, and use the time and frequency metrology as fundamentals in the navigation service.
\end{abstract}

Keywords: Atomic clock, Time scale, Time measurement, Navigation, Timekeeping, UTC

\section{Introduction}

Navigation and timekeeping have always been strongly related. The current GNSSs are based on a strict timekeeping system and the core measure, the pseudo-range, is actually a time measurement. To this aim, very good clocks are installed on board GNSS satellites, as well as in the ground stations and control centres.

National and international timekeeping relies on GNSS. In fact, the international reference time, Coordinated Universal Time (UTC), is obtained from the average of about 450 atomic clocks maintained in about 80 national laboratories worldwide, and the clocks of the different laboratories are generally compared by means of GNSS.

In this case, instead of using GNSS to estimate position, the time transfer users mostly use the timing information provided by GNSSs and, in some cases, they don't solve for their position as it is already known with sufficient accuracy.

Moreover, the current GNSS not only offer positioning services, but also timing services as they broadcast suitable information on UTC and the user can obtain timing

${ }^{*}$ Correspondence: patrizia.tavella@bipm.org

Bureau International des Poids et Mesures, Pavillon de Breteuil 92312 Sèvres Cedex, France information. This is accomplished by a precise connection between the GNSS control centre and some of the national laboratories that participate to UTC and realize their real-time local approximation of UTC.

These features are reviewed in this paper offering an overview of the mutual advantages between navigation and time keeping, particularly the cross-fertilization that has always been achieved in these two fields.

\section{Clocks and time metrology in GNSS}

In modern GNSS, the position of the user is estimated as the intersection of three or more spheres whose centre is the known position of a satellite and whose radius is given by the velocity of light multiplied by the travel time of the satellite signal, knowing when it started and measuring, by the local receiver, when it arrived.

Since the light velocity is a very large number, it is sufficient to allow an error of $1 \mathrm{~ns}\left(10^{-9} \mathrm{~s}\right)$ to obtain at least $30 \mathrm{~cm}$ of error in the estimated position. A time error of one nanosecond between a space-based to ground clock is difficult to achieve; an error of $100 \mathrm{~ns}$ is more common, which would give a positioning error of 30 metres. This gives an understanding of why time metrology is vitally important in navigation.

With reference to Fig. 1, we see that all the segments of a GNSS are equipped with clocks, with different 


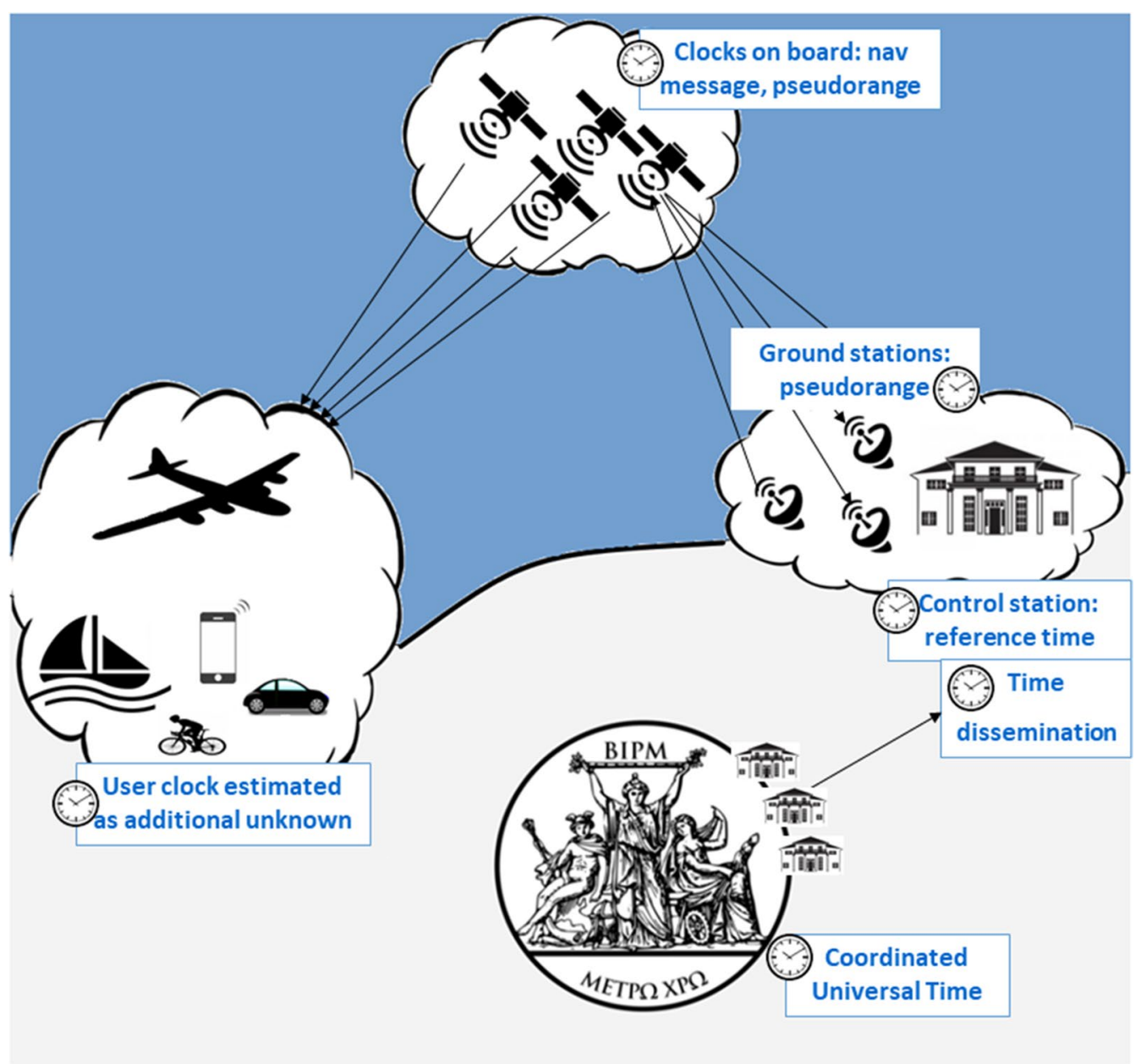

Fig. 1 The typical GNSS structure and the presence of clocks

purposes. The more stable, reliable, and space-qualified atomic clocks have the aim of keeping time onboard satellites and to generate the navigation signal.

On the ground, the network of monitoring stations, as well as the control centres, need stable and accurate clocks to generate the system reference time, to measure the satellite pseudo-ranges, and to ensure the orbit and clock estimation and prediction.

User receivers are equipped with clocks, to measure the time of arrival of the satellite signals. The receiver clock, not necessarily an atomic clock, has an unknown offset which needs to be estimated in the whole navigation solution, adding a fourth unknown to the estimation problem, besides to three space positioning coordinates.

In principle, this is sufficient to estimate the user's position, but to offer an additional timing service, the GNSSs are in some way connected to time laboratories that realize local approximations of Coordinated Universal Time (UTC), the ultimate reference time realized by the
Bureau International des Poids et Mesures (BIPM) [https ://www.bipm.org/en/bipm/tai/].

If we write the typical pseudo-range equation, we can immediately see how time metrology is imbedded in the navigation solution (see for example Tavella 2017).

If we indicate by $\mathbf{x}_{r e c}$ the receiver's unknown 3-dimensional position, similarly $\mathbf{x}_{\text {sat }}$ indicates the 3-dimensional satellite position. If $t_{s a t}$ is the epoch at which the signal leaves the satellite, and $t_{r e c}$ is the epoch at which it arrives at the receiver, the distance between satellite and receiver is indicated by Eq. (1)

$$
D_{r e c}^{s a t}=c\left[\left(t_{r e c}-t_{s a t}\right)\right]=\left|\mathbf{x}_{s a t}-\mathbf{x}_{r e c}\right|
$$

where $c$ is the velocity of light. The satellite position and epoch of transmission are known, as transmitted by the same satellite in the navigation message, the epoch of reception is measured, therefore the unknowns are the 3-dimensional space coordinate $\mathbf{x}_{\text {sat }}$ 
and by measuring three satellites the system could be resolved. This is true in principle but what happens if a user's measurement is actually a pseudo distance, or pseudo range, which contains other unwanted contributions. A complete equation could be

$$
\begin{aligned}
P_{r e c}^{s a t}= & c\left[\left(t_{r e c}-t_{\text {sat }}\right)\right]=\left|\mathbf{x}_{\text {sat }}-\mathbf{x}_{\text {rec }}\right| \\
& +c\left[\left(t_{r e c}-t_{r e f}\right)-\left(t_{\text {sat }}-t_{r e f}\right)\right]+I_{r e c}+T r+\varepsilon_{r e c}
\end{aligned}
$$

where we find more terms. Firstly, the fact that the system and the receiver clocks are not perfectly synchronized should be taken into account, they may have an offset with is indicated with respect to a certain common reference time indicated by $t_{\text {ref }}$. The receiver clock offset is actually introduced as a fourth unknown of the system for which we are asking for a fourth satellite to be measured.

The offset $\left(t_{\text {sat }}-t_{\text {ref }}\right)$ between the satellite and the reference time takes into account the fact that the clock onboard can have an offset and this offset is to be communicated to the user to be taken into account in the navigation solution. The terms $I_{r e c}, T r$, and $\varepsilon_{\text {rec }}$ are taken into account additional delays due to the ionosphere, the troposphere and the receiver hardware.

If we concentrate only on the timing contribution, from (Eq. 2), we see some important issues:

1. The reference time $t_{\text {ref }}$ is the navigation system Reference Time to be defined from the ensemble of space/ ground clocks, similarly to any national reference time scale. For example: (a) GPS time is a paper time scale estimated with a Kalman filter and steered versus UTC(USNO),

(b) Galileo System Time is a weighted average of the ground clocks steered versus UTC,

2. The offset $\left(t_{s a t}-t_{r e f}\right)$ is estimated by the algorithm that processes the same pseudorange measures and estimating orbits and clocks (Kalman filter in case of GPS, batch least square in case of Galileo, ...)

3. The real time offset $\left(t_{s a t}-t_{r e f}\right)$ transmitted to the user is actually a prediction based on previous measures, the prediction method depends on the type of clocks, the latency, and the requested uncertainty.

The information on the satellite position and the onboard clock are communicated to the satellite by the ground control segment and they are estimated with previous pseudo-range measures obtained by the network of ground monitoring stations. What is uploaded to the satellite can only be a prediction to be transmitted in the navigation message to the users. The predicted satellite clock offsets (and orbits) need to be valid for a certain period in the future, until the satellite is again in view of the uploading station to receive fresh estimates.

Clock measurement, estimation and prediction, as the definition of the reference time and its steering versus UTC are the typical activities of a timekeeping laboratory. The clocks and the requirements may be different, but very similar issues are faced. For a general description of GNSS many books and papers are available, for example (Parkinson and Spilker 1996). An overview of

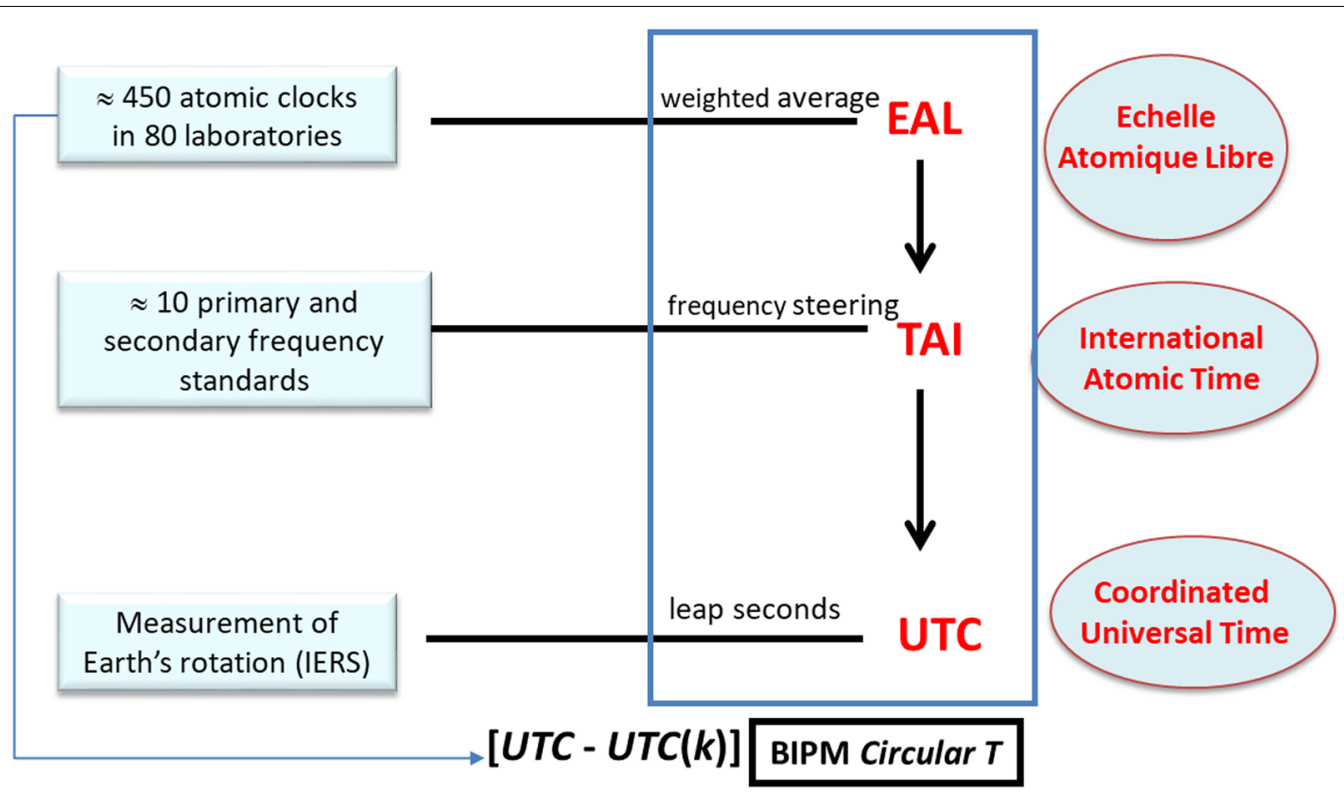

Fig. 2 The computation scheme of UTC (Panfilo and Arias 2019) 
time metrology issues in GNSS examples can be found in Sesia et al. (2014).

\section{GNSS in international timekeeping and in Coordinated Universal Time}

Keeping time, in navigation and in metrology, requires suitable clocks and primary frequency standards, clock measuring equipment in the laboratory and at distance, appropriate algorithms for data processing. Finally, it requires excellent international coordination as in the case of UTC. In this section we describe the activities necessary to build the international reference time scale UTC (Coordinated Universal Time), which is carried out at the Bureau International des Poids et Mesures (BIPM). We concentrate on the description of the role of GNSS measures in UTC. A recent overview of UTC is available explaining the details of UTC computation and the necessary measurements (Panfilo and Arias 2019). The general scheme of computation of UTC is reported in Fig. 2.

There are about 450 atomic clocks maintained in almost 80 national metrology institutes or observatories all around the world that are used to compute a type of "weighted average" that is referred to in French as the "Echelle Atomique Libre", i.e. free atomic time scale. The distribution of the UTC laboratories as well as their main time transfer system is illustrated in Fig. 3.

The necessary input for this computational step is the measurement of the offset between all clocks. Clocks inside the same laboratory are measured by appropriate electronic devices as counters or phase comparators, while the comparison from a time laboratory to another requires special systems, and this is where GNSSs play a role.

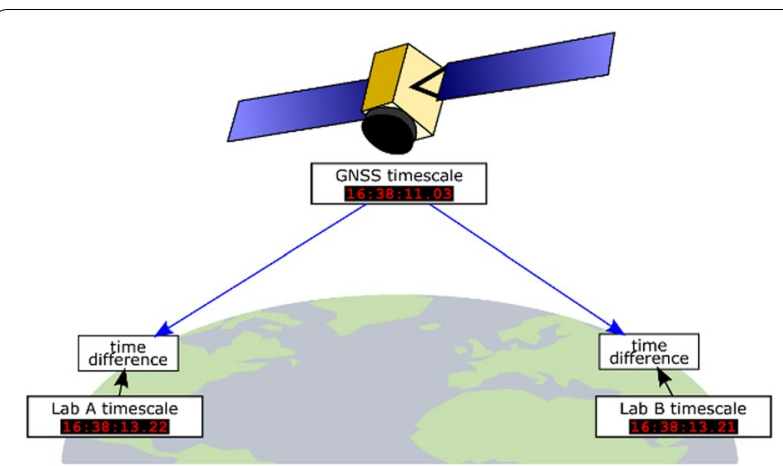

Fig. 4 Principle of the GNSS time transfer

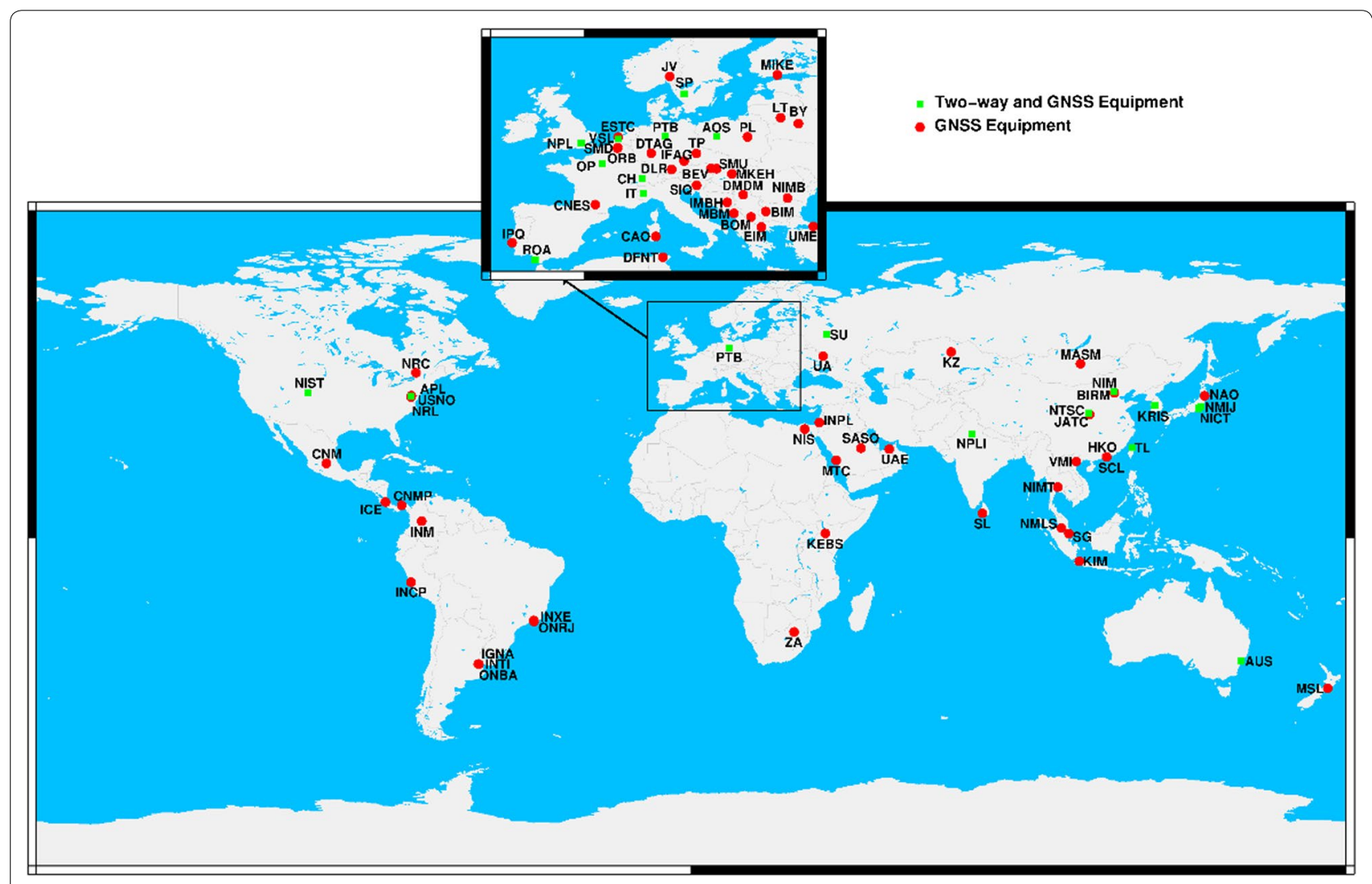

Fig. 3 Geographical distribution of the laboratories contributing to UTC (2018) 
Table 1 Example of CGGTTS file with the GNSS measures

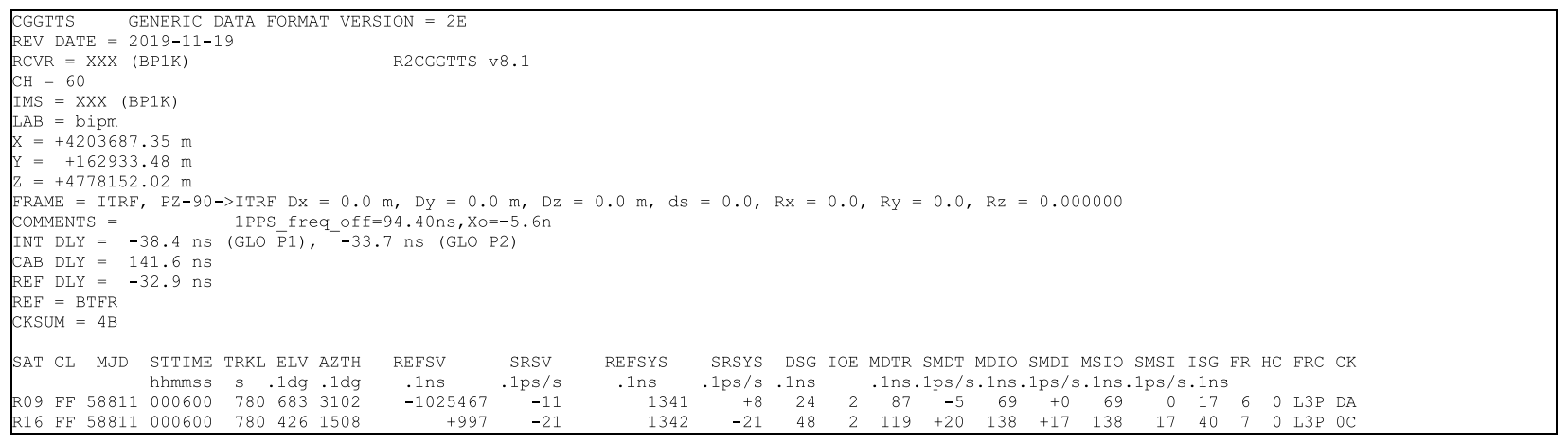

The GNSS can in fact be used as "time comparison" technique: knowing the receiver position and the information contained in the GNSS navigation message, the pseudorange Eq. (1) can be solved to estimate the receiver clock offset. Different time comparison techniques based on GNSS measures have been developed and they are still fostered with the aim to improve the achievable accuracy.

\section{Clocks compared by GNSS (plus other techniques)}

The basic clock comparison technique by means of GNSS is based on the code pseudo-range measures by two receivers (Fig. 4) and observation can be in Common View or All in View.

\section{GNSS common view and all in view}

The common-view ( $\mathrm{CV}$ ) method relies on reception by several receivers of the same emitted signal, with the drawback that, with increasing distances, the number of simultaneously observed satellites decreases, and the number of high elevation satellites becomes low. GPS CV had been used for the calculation of UTC at the BIPM until 2006. It is still in use for clock comparisons because it is very simple to operate, particularly over short distances, when it is sufficient to mitigate the error from satellite orbits. Using a special time receiver, the user has the possibility to obtain a clock comparison resolved by the receiver and which is available in an output file according to the CGGTTS format (Allan and Thomas 1994; Defraigne and Petit 2015). An example of the CGGGTT $\mathrm{S}$ file is shown in Table 1 . The header contains information on the GNSS receiver as well as its calibration; the data columns contain identification of the date, satellite, orbit parameters, and timing information. For example, the 8th column "REFSV" indicates the offset "REFerence user clock-Satellite Vehicle clock", i.e. the offset between the user clock and a satellite clock, while the 10th column "REFSYS" contains the offset "REFerence user clock
- SYStem time scale", i.e. the offset between the user clock and the GNSS system time.

Advances in obtaining precise satellite orbits and clock parameters allowed the introduction of another technique, known as All in View (AV) (Petit and Jiang 2008), where all satellites in view are used to compare the local clock to a reference time scale through the satellite clocks. AV eliminates the constraint of simultaneous observations, so that two observing stations can be linked whatever their distance. The International GNSS Service (IGS) has provided, since 2004, high-precision GPS satellite clock products referred to a common time scale (Dow et al. 2009) in addition to precise satellite orbits. This made it possible to use the AV method instead of the CV method, resulting in a statistical uncertainty of the time links independent of the distance. The AV method has been used for all UTC links since the end of 2006. Over the years, most GPS receivers have become dual-frequency, providing ionosphere-free data, denominated GPS P3, and allowing sub-nanosecond statistical uncertainty for the links.

\section{GNSS precise point positioning (and IPPP)}

With code-only techniques, multipath effects and errors in the tropospheric model, together with code noise, ultimately limit the performance either with $\mathrm{CV}$ or AV. The addition of phase measurements from geodetic-type receivers minimizes the effects of these error sources (Dach et al. 2002; Ray and Senior 2003, 2005). The precise point positioning technique (PPP) (Kouba and Heroux 2001) in which dual frequency phase and code measurements are used to compare the reference clock in a station to a reference time scale, is now the basis of GNSS time comparisons worldwide. It has been implemented for the computation of UTC time links since September 2009 (Petit and Jiang 2008). By this technique the statistical uncertainty of clock comparison is about $0.3 \mathrm{~ns}$ for any averaging time up to 1 month. 
For frequency comparisons, PPP provides accuracy in the low $10^{-16}$ although this is not sufficient to compare the present primary and secondary frequency standards. One main limiting factor of PPP comes from the effect on the clock solution of the resolution of real-valued phase ambiguities together with other parameters. To overcome this limitation, techniques have been developed to consider the integer nature of phase ambiguities in the solution. The IPPP technique (Petit et al. 2015) allows frequency comparisons with $1 \times 10^{-16}$ accuracy, typically within a week of averaging and reaches the low $10^{-17}$ range in 20-30 days.

\section{Multi GNSS in UTC}

Many laboratories contributing to UTC operate multiGNSS receivers, allowing the computation of time links using not only GPS but also the other GNSS such as
GLONASS, Galileo and BeiDou, which have all reached operational status.

A few GLONASS time links, as well as combinations of GPS and GLONASS links, have been used in UTC for many years (Jiang and Lewandowski 2012). However, the use of GLONASS is hindered by problems in the calibration due to the use of different frequencies for different satellites. A recent study (Petit and Harmegnies 2019) has shown that Galileo can provide dual frequency code links with an uncertainty at least as good as GPS. As an example Fig. 5 shows a very long link between Singapore and Brussels with a noise level below $0.5 \mathrm{~ns}$ at $1000 \mathrm{~s}$ averaging time for both Galileo and GPS. The same study, conducted with the BDS-2 constellation, concluded that such dual frequency code links can achieve a noise level about similar to GPS only in the East-Asia region (see Fig. 5). For links involving stations outside East-Asia, the uncertainty is somewhat

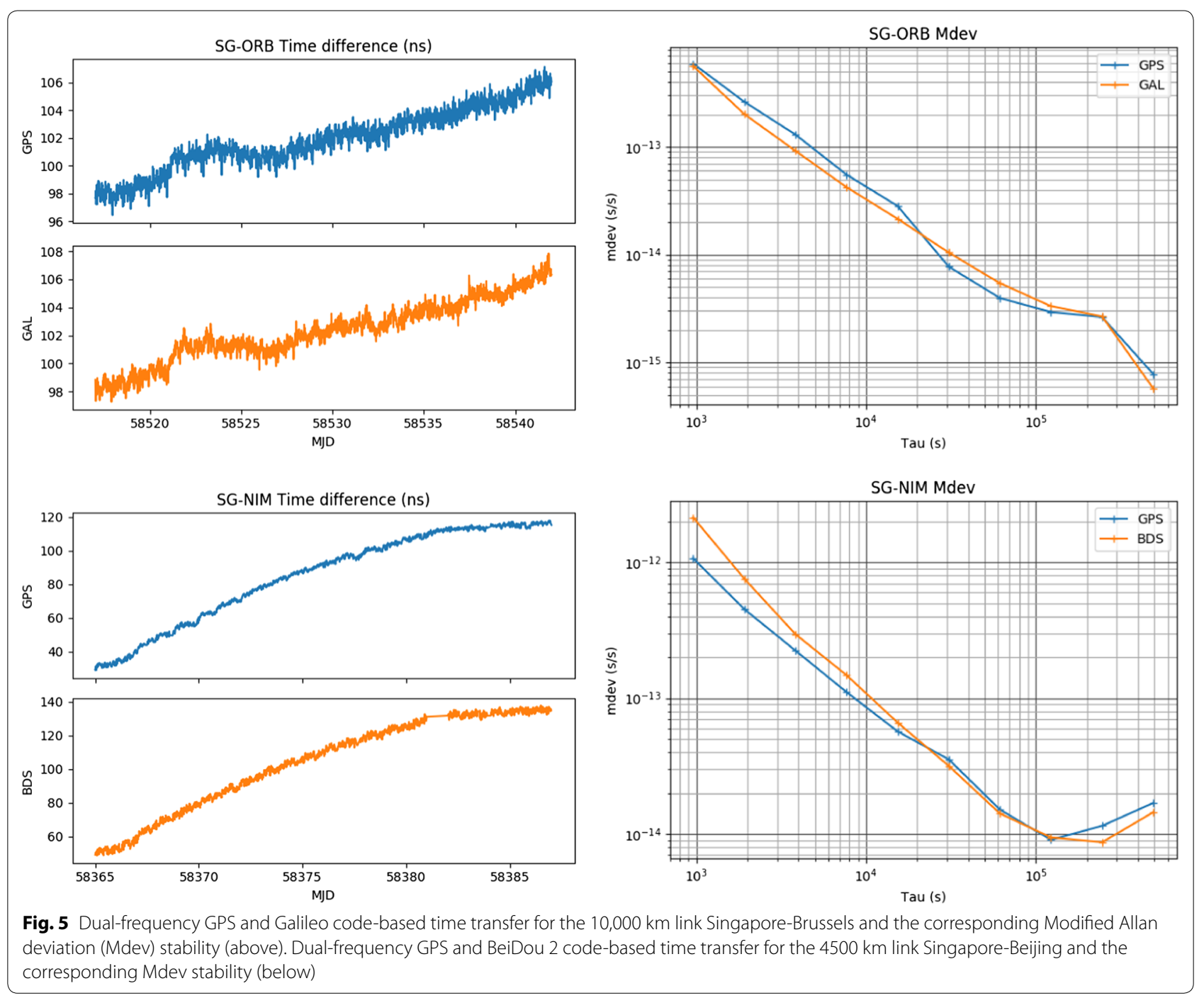




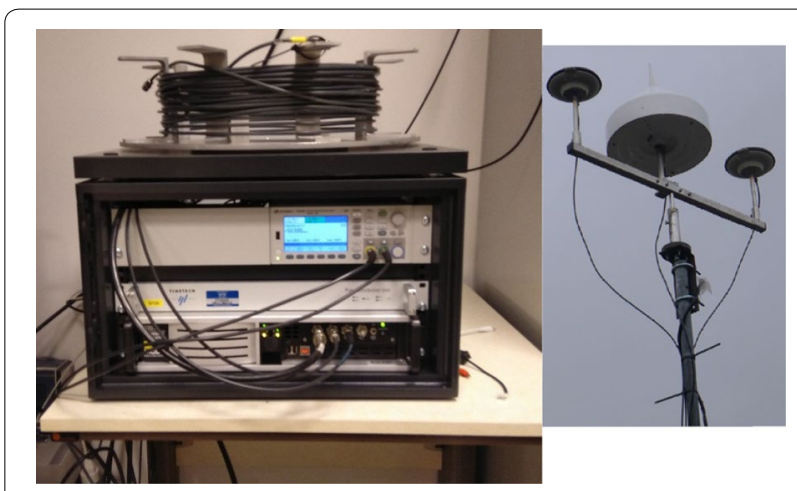

Fig. 6 BIPM new travelling GNSS station for calibration

degraded due to the low number of available measurements, a situation that should improve with the BDS-3 constellation. Similar results were found for BeiDou by Liang et al. (2018). In a more extended study including all four GNSS (Verhasselt and Defraigne 2019) obtained similar conclusions, but they also considered various methods to combine the data from several constellations and identified some combinations that improve the time transfer performance with respect to any single-GNSS solution. The optimal use of all GNSS, either as a single system or in a multi-system combination is still under investigation.

\section{GNSS receiver calibration for UTC}

The accuracy of the GNSS time transfer techniques largely depends on the calibration of the receivers. Since 2014, the calibration of receivers contributing to UTC has been organized in two levels: the BIPM regularly calibrates a small network of "Group 1" laboratories in each region of the world; those laboratories then calibrate the remaining laboratories. The calibration procedure and results are available at https://www.bipm.org/jsp/en/ TimeCalibrations.jsp. Figure 6 shows the new BIPM calibration travelling equipment.

\section{Other clock comparison techniques (not based on GNSS)}

GNSS techniques based on commercial receivers are widely used in most time laboratories, but other techniques have been developed and are used. This ensures independent comparisons and possible improvements in accuracy.

The main techniques are known as Two Way Satellite Time and Frequency Transfer (TWSTFT) (Kirchner 1991), where a dedicated ground terminal simultaneously receives and transmits time transfer signals on geostationary satellites. In this scheme, the propagation time of the signal is cancelled (at first order).
Recently the TWSTFT technique has been enhanced through the use of a Software Designed Radio Receiver (Jiang et al. 2018) and new transmitting systems are under development, which are based on the carrier phase.

The most promising time and frequency transfer for the future is based on optical fibres and these networks are under development in different regions (see Calonico et al. 2015; Lisdat et al. 2016; Riehle 2017).

\section{The computation of the international atomic time}

After the first step of clock averaging, some other special clock measures are inserted. Some metrological laboratories have developed and operate high-accuracy frequency standards, where the quantum transition of the caesium atom that realizes the second is directly generated by observing the atoms and evaluating all the possible perturbation effects. These are called primary frequency standards. Usually these standards do not operate continuously as clocks, as they are very complex instruments, therefore they are used intermittently to calibrate the frequency of EAL. Similar standards based on other atoms are also recognized as "secondary" representations of the seconds (BIPM Web Page 2018), therefore primary and secondary frequency standards (PFSF) are used to evaluate the residual frequency offset of the ensemble average EAL. This residual offset is compensated with very small frequency steps (recently $2 \times 10^{-16}$ in relative value) and the steered time scale is called International Atomic Time (TAI, in French). Figure 7 reports the calibration of EAL frequency by PSFSs since 2006.

With the stability optimization obtained by the weighted average and the accuracy obtained by comparison with PSFS, TAI now has an accuracy of about $2 \times 10^{-16}$ in relative value, and it represents the international standard for time and frequency metrology.

\section{Keeping pace with the Earth's rotation}

The duration of the atomic second was defined in 1967 and is in agreement with the previous definitions of the second based on Earth rotation. However, the Earth has irregularities in its motion and it is possible to observe a slight slowing down over the centuries. This means that timekeeping based on the rotation of the Earth is slowly divergent with respect to the most precise timekeeping based on atomic clocks.

When TAI was defined in the 1970s, it was deemed important to have a civil timekeeping reference that was still based on the rotation of the Earth as, during that epoch, most marine navigators needed to know the rotational angle of the Earth to determine their position at sea. It was therefore decided to realize a modified version 


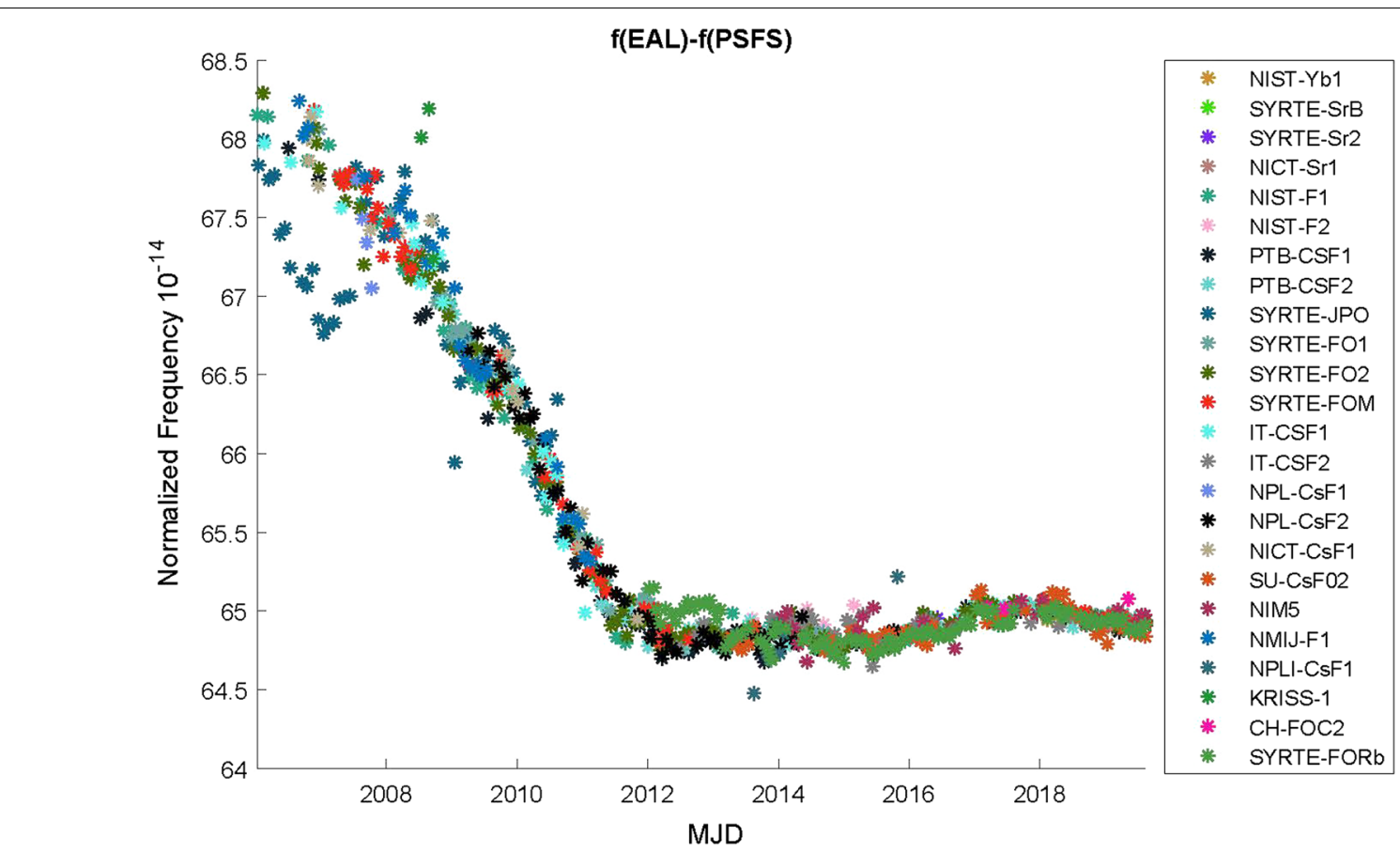

Fig. 7 Measures of the EAL frequency offset versus primary and secondary standards. The code of the standard is reported in the legend (see BIPM Annual reports https://www.bipm.org/en/bipm-services/timescales/time-ftp/annual-reports.html)

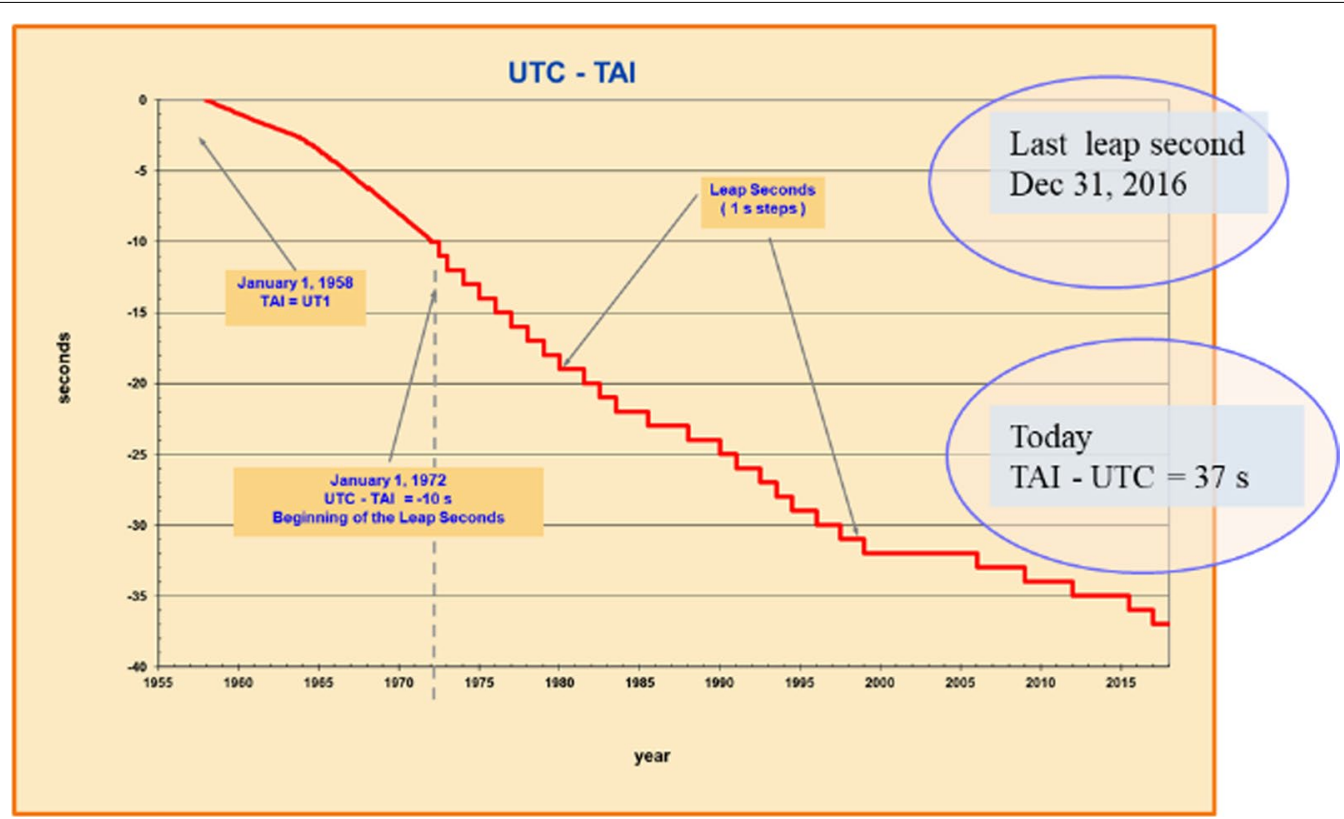

Fig. 8 The Universal Time Coordinated and its steps from TAI. The insertions of leap seconds started in 1972

of TAI, named UTC, with the same quality of an atomic time scale, but with the insertion of a leap second so that at any time, the divergence between UTC and the Earth's rotational angle was smaller than a second. The offset between UTC and TAI is illustrated in Fig. 8 .

Nowadays, the management of highly technological and complex systems such as GNSS, telecommunication 


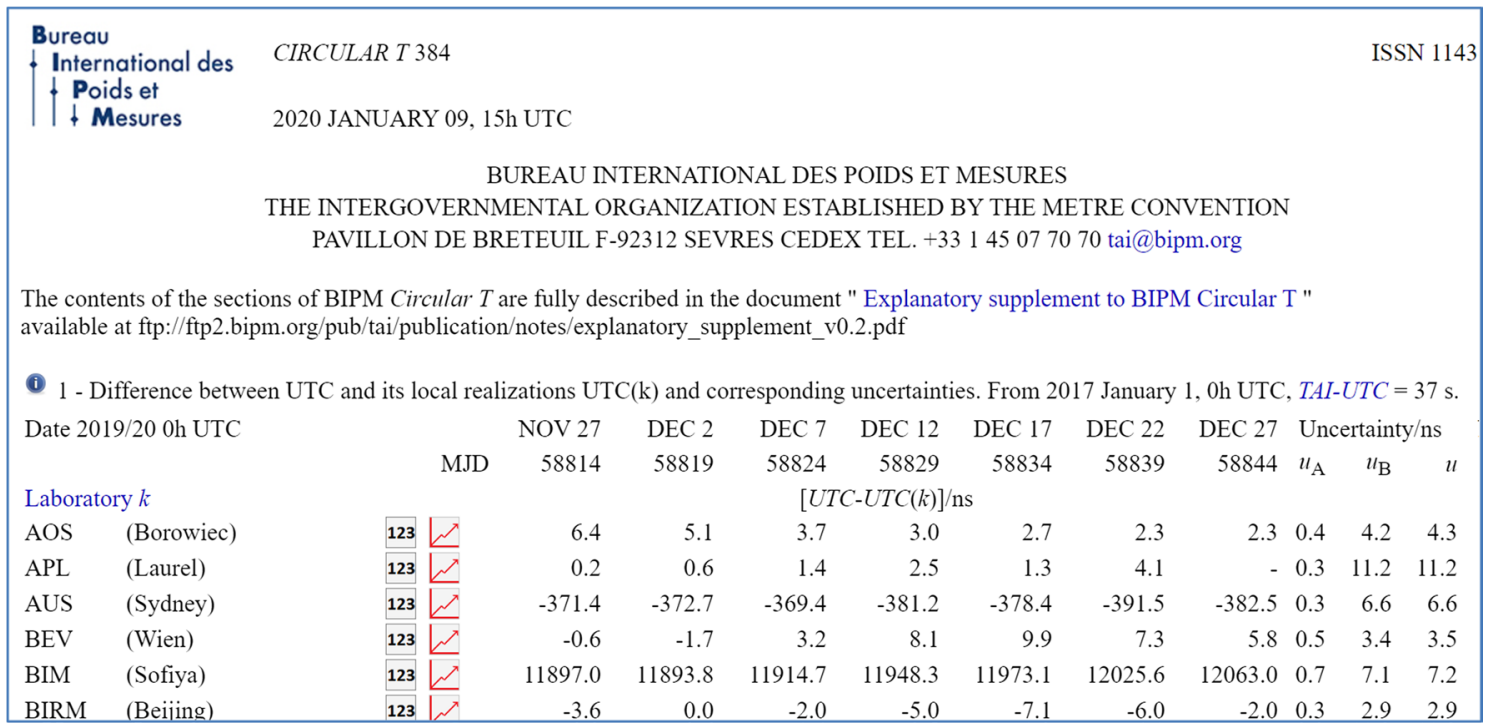

Fig. 9 An extract from the BIPM CircularT of Dec 2019 showing the differences UTC-UTC(k) on standard dates separated by 5 days. Details can be found here (https://www.bipm.org/en/bipm-services/timescales/time-ftp/Circular-T.html)

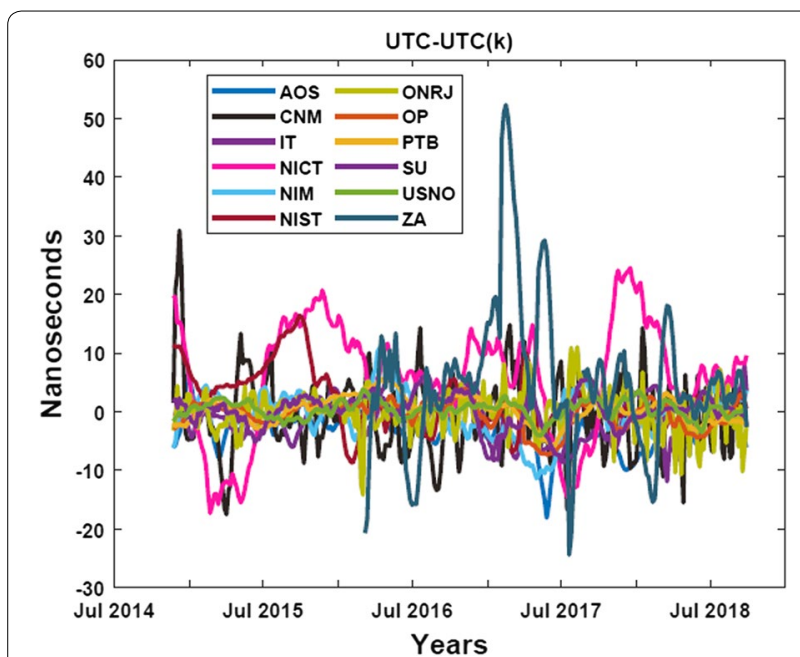

Fig. 10 Examples of the offset of some UTC(k) time scales versus UTC in the last years

or data transfer networks is mostly based on continuous time scales without any abrupt insertion of a leap second, therefore the advantage of a reference time scale in agreement with the rotation of the Earth, such as UTC is put into question. On the definition of UTC, the related benefit and drawbacks, the reader is referred to a special issue of Metrologia (McCarthy 2011).

\section{Publishing UTC results}

The computation of UTC is under the responsibility of the BIPM (www.bipm.org), while the insertion of leap seconds is announced by the IERS, the International
Earth Rotation and Reference Systems Service www.iers. org.

Every month, the BIPM publishes the results of UTC computation in a bulletin called "Circular $T$ " and they are made available on the BIPM web page as well as in a dedicated data base (http://webtai.bipm.org/datab ase/) accessible to all users. An extract from Circular $T$ is shown below (Fig. 9).

The results report the offset between UTC and each local approximation realized in laboratory " $k$ ", named $\mathrm{UTC}(k)$. Universal Coordinated Time is the ultimate time reference available in deferred time, while local time scales $\mathrm{UTC}(k)$ are realized by national laboratories in real time. A posteriori, they are verified versus UTC. Examples of the offset of some national time scales $\mathrm{UTC}(k)$ versus UTC are shown in Fig. 10.

The BIPM also computes a rapid solution for UTC which is indicated by UTCr, this is computed every week, based on daily data (Petit et al. 2014). Results are available here https://www.bipm.org/en/bipm-services/times cales/time-ftp/Rapid-UTC.html.

\section{UTC dissemination by GNSS}

GNSS systems, developed for navigation and positioning, have added an additional time dissemination service by estimating, predicting, and transmitting the offset between the GNSS time scale and UTC in their navigation message. In each system, all the clocks are kept synchronized to a system time scale, as the GPStime or the GLONASStime, for example. We can refer to GNSST as this system time. 
From the navigation solution, the user can have access to the estimate of the offset of his/her receiver clock with respect to the GNSS system time. This is, for example, one of the types of information reported in the CGGTTS file in the 10th column named REFSYS (Table 1), standing for "REFerence user clock - SYStem time scale", which can be referred to more simply as "User clock - GNSST" for this quantity.

Knowing the offset of the GNSS system time versus UTC from the navigation message, the user can get an estimate of the offset of his/her clock with respect to UTC. In the case of GPS, for example, the navigation message is transmitting the offset of GPS time versus UTC(USNO), where USNO in the US Naval Observatory. The user can obtain

$$
\begin{aligned}
& \text { User clock - UTC(USNO) } \\
& =(\text { User clock }- \text { GPStime })_{\text {nav solution }} \\
& \quad+(\text { GPS time }- \text { UTC }(\text { USNO }))_{\text {nav message }}
\end{aligned}
$$

In this case the information transmitted by GPS is based on a special realization of UTC from the USNO, named UTC(USNO) (Interface Specification 2018). In the case of GLONASS, the transmitted UTC information is UTC(SU), as realized by the Russian metrological laboratory VNIIFTRI (Interface Control Document 2008). The more recent GNSSs, BeiDou and Galileo, transmit a prediction of the offset of their time scale with reference to UTC, based on the measures versus some local

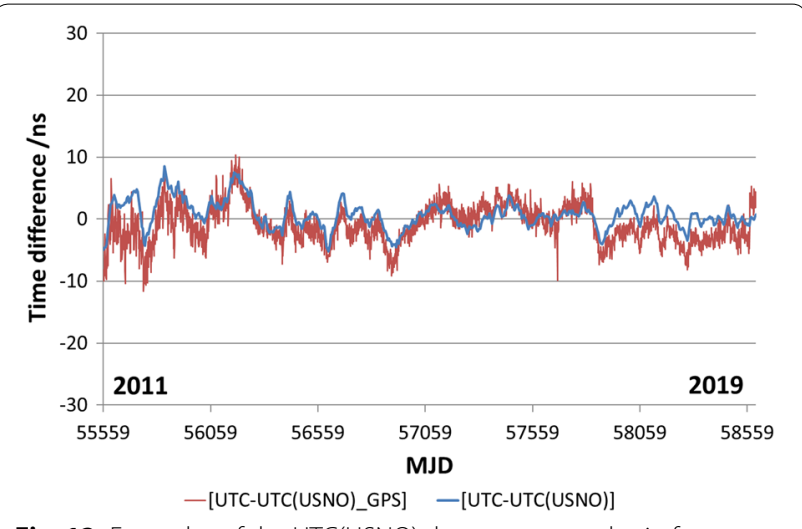

Fig. 12 Examples of the UTC(USNO) that a user can obtain from the information broadcast by GPS with respect to the UTC(USNO) estimated by the BIPM and published in Circular T

$\mathrm{UTC}(\mathrm{k})$ time scales in China and Europe, respectively See (BeiDou ICD 2019; Galileo 2019).

It is important to mention that in this case the calibration of the user receiver is fundamental, as the delay introduced by the antenna, the cables, and the receiver are to be carefully measured to be able to connect to UTC without any unknown bias.

For the purpose of information for the users and to the GNSS providers, the BIPM publishes an estimate of the offset between the BIPM UTC and the proxy UTC broadcast in the GNSS navigation message in Circular T. As

4 - Relations of UTC and TAI with predictions of UTC(k) disseminated by GNSS.

[UTC-UTC(USNO)_GPS] $=\mathrm{C}_{0}{ }^{\prime}$, [TAI-UTC(USNO)_GPS] $=37 \mathrm{~s}+\mathrm{C}_{0}{ }^{\prime}$

[UTC-UTC(SU)_GLONASS] $=\mathrm{C}_{1}{ }^{\prime},\left[\right.$ TAI-UTC(SU)_GLONASS] $=37 \mathrm{~s}+\mathrm{C}_{1}{ }^{\prime}$

For this edition of Circular $T, \sigma_{0}^{\prime}=0.8 \mathrm{~ns}, \sigma_{1}^{\prime}=6.7 \mathrm{~ns}$

$\begin{array}{lccccc}2019 & \text { M h UTC } & \mathrm{C}_{0}{ }^{\prime} / \mathrm{ns} & \mathrm{N}_{0} & \mathrm{C}_{1}{ }^{\prime} / \mathrm{ns} & \mathrm{N}_{1}{ }^{\prime} \\ \text { MAR 27 } & 58569 & -4.8 & 89 & 10.1 & 80 \\ \text { MAR 28 } & 58570 & -0.6 & 72 & 10.3 & 88 \\ \text { MAR 29 } & 58571 & 4.2 & 90 & 9.8 & 89 \\ \text { MAR 30 } & 58572 & 3.4 & 89 & 8.7 & 77 \\ \text { MAR 31 } & 58573 & 3.5 & 89 & 11.0 & 86 \\ \text { APR 1 } & 58574 & 2.3 & 89 & 11.7 & 80 \\ \text { APR 2 } & 58575 & 1.9 & 90 & 8.0 & 60 \\ \text { APR 3 } & 58576 & 2.9 & 86 & 7.0 & 78\end{array}$

Fig. 11 Examples of the offset of UTC versus the UTC information broadcast by GPS and GLONASS in BIPM circular T 


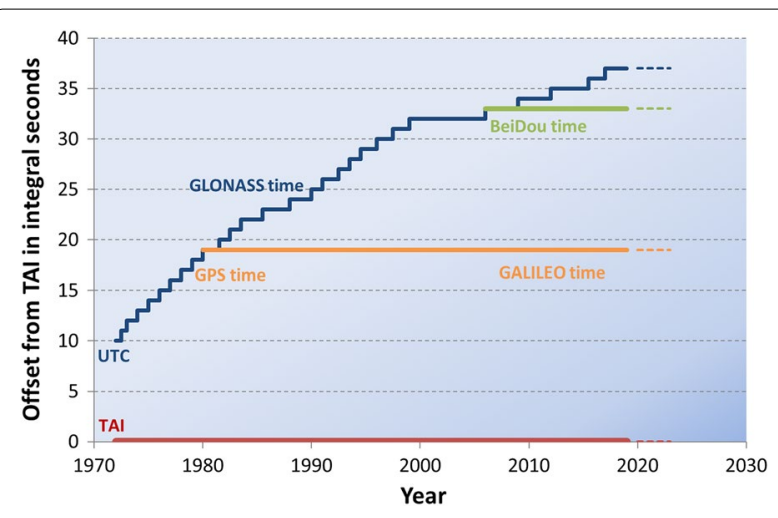

Fig. 13 Offset of GNSS system time scales and UTC versus TAI due to the introduction of leap seconds

reported in Fig. 11, Circular T section 4 reports, for each day of the month, the quantity

$$
\begin{aligned}
& \text { UTC - UTC(USNO)_GPS and } \\
& \text { UTC - UTC(SU)_GLONASS }
\end{aligned}
$$

where UTC(USNO)_GPS stands for the UTC(USNO) time scale as disseminated by GPS, and similarly for UTC(SU). These quantities are computed at the BIPM based on calibrated receivers placed in UTC laboratories. The BIPM plans to add additional information on the UTC dissemination service by BeiDou and Galileo systems.

To have an idea of the quality of the UTC broadcast information, for example by GPS, in Fig. 12 we can see the offset UTC - UTC(USNO) as computed by the BIPM as part of the Circular $T$ results, and a similar quantity denoted by UTC-UTC(USNO)_GPS where the quantity UTC(USNO)_GPS is obtained by the BIPM as explained before (Fig. 11). It can be seen that over a period of several years the time scale UTC(USNO) as estimated, predicted, and broadcast by the GPS system is in very good agreement, at the level of a few nanoseconds, with respect to the "true" UTC(USNO) estimated by the BIPM and reported in BIPM Circular T. GPS users therefore have very good information on UTC(USNO) by GPS in real time all around the world.

We will now return to the leap second procedure described in section "Keeping pace with the Earth's rotation". GNSS management systems, with the exception of GLONASS, prefer not to apply leap seconds to their system time scale in order to avoid issues during the operation of the system with steps in the reference time scale. Therefore, at a certain initial moment the GNSS system time is synchronized to UTC, but then the subsequent leap seconds are no longer applied and the GNSS time scale gains a growing offset with respect to UTC at the level of seconds. This is illustrated in Fig. 13 where the offset of UTC and the GNSS time scales versus TAI, the International Atomic Time, is reported.

The fact that GNSS system times have different methods of counting seconds with respect to UTC should not trouble the user as the correct timing information from the GNSS navigation message is the information concerning the disseminated UTC and not the GNSS system time scale. Nevertheless, the availability of the GNSS system times that are easily accessible from a GNSS receiver in real time and worldwide can generate some confusion. This is one of the reasons for which the current practice of using leap seconds was called into question several years ago and still is under debate

\section{Can UTC help GNSS interoperability?}

Finally, we will discuss the possible benefit of the UTC time scale for interoperability of different GNSSs.

The International Committee on Global Navigation Satellite Systems (ICG) (https://www.unoosa.org/oosa/ en/ourwork/icg/icg.html), of the United Nations, had formerly initiated a discussion on the need for a common reference time scale for all GNSSs to be used to ensure interoperability of different GNSSs. In fact, as the GNSS system times are different for each system, the user needs to get the information on the offset between such system's times or the receiver needs to estimate them as additional unknowns in the navigation solution. Recent studies show that in good visibility conditions it is always a better solution for the user's receiver to estimate those offsets, but in the case of very poor visibility it may be useful to have additional broadcast information. The main proposal is that each GNSS could transmit the offset of its GNSS system time with respect to a unique common reference time, and the users could then estimate all the intersystem offsets.

There are different possibilities to create or retrieve such a unique reference time. The BIPM and the metrological community investigated the possibility of using UTC as a common reference time scale that is already available and already estimated by all GNSSs. From experimental tests (Signorile 2019; Defraigne 2020) it appears that the contribution to the uncertainty in timing and positioning of a MultiGNSS user, due to the fact that the UTC proxy used by the different GNSSs are not the same and the prediction/estimation techniques are also different, amounts to a few nanoseconds (for example 1-2 m), which is acceptable in conditions of poor visibility where the error in positioning and timing is, in any case, larger than that.

In a recent meeting the International Committee for Weights and Measures, which is working to ensure 
worldwide uniformity in units of measurement, adopted the following decision:

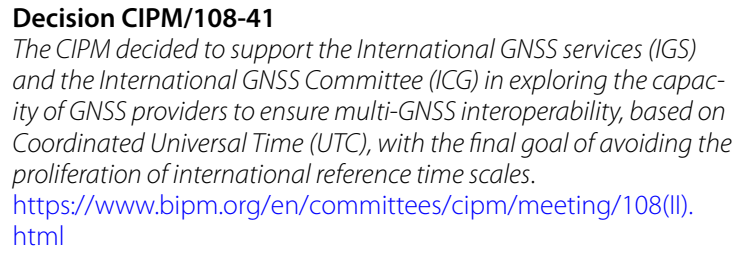

\section{Conclusions}

Interactions between timing and positioning have always existed for example through the need of precise clocks to obtain the longitude at sea. These interactions are stronger than ever with the advent of atomic clocks and GNSS.

This liaison has created a great cross-fertilization and has allowed a strict cooperation among scientists and engineers from both fields. The current GNSS management systems strictly collaborate with UTC time laboratories and are corroborating this fruitful relationship.

\section{Acknowledgments}

The support and contribution of the colleagues from the BIPM Time Department as well as all the discussion with the colleagues in the time metrology and in the navigation communities are kindly acknowledged.

\section{Authors' contributions}

PT proposed the idea and prepared the main review, while GP contributed to the "Clocks compared by GNSS (plus other techniques)" section as leading expert of GNSS time transfer in UTC. Both authors read and approved the final manuscript.

\section{Funding}

Not applicable.

\section{Availability of data and materials}

The used data showed in the plots are obtained from the BIPM web site and they are duly referenced in the text.

\section{Competing interests}

The authors declare that they have no competing interests.

Received: 11 December 2019 Accepted: 25 February 2020

Published online: 16 March 2020

\section{References}

Allan, D. W., \& Thomas, C. (1994). Technical directives for standardization of GPS time receiver software. Metrologia, 31, 69-79.

BeiDou ICD, Signal in Space Interface Control Document. (2019). http://m. beidou.gov.cn/xt/gfxz/201902/P020190227593621142475.pdf.

BIPM Web Page. (2018). https://www.bipm.org/en/publications/mises-en-prati que/standard-frequencies.html.

Calonico, D., Inguscio, M., \& Levi, F. (2015). Light and the distribution of time. EPL, 110, 40001

Dach, R., Hugentobler, U., Schildknecht, T., Bernier, L. G., \& Dudle, G. (2002). Precise continuous time and frequency transfer using GPS carrier phase. IEEE Transactions on Ultrasonics, Ferroelectrics, and Frequency Control, 49(11), 1480-1490.

Defraigne, P., et al. (2020). "Impact of inter-system biases accuracy on multi-GNSS positioning and timing", to be presented at the European Frequency and Time Forum, Noodwijk, 2020
Defraigne, P., \& Petit, G. (2015). CGGTTS-Version 2E: An extended standard for GNSS. Metrologia, 52, 6 G1.

Dow, J. M., Neilan, R. E., \& Rizos, C. (2009). The international GNSS service in a changing landscape of Global Navigation Satellite Systems. Journal of Geodesy, 83(3-4), 191-198.

Galileo SDD, Open Service - Service Definition Document. (2019). www.gsceuropa.eu/system/files/galileo_documents/Galileo-OS-SDD.pdf.

GLONASS ICD, Interface Control Document. (2008). http://gauss.gge.unb.ca/ GLONASS.ICD.pdf.

GPS ICD, Interface Specification. (2018). https://www.gps.gov/technical/icwg/ IS-GPS-200J.

Jiang, Z., \& Lewandowski, W. (2012). Use of GLONASS for UTC time transfer. Metrologia, 49, 57-61.

Jiang, Z., Zhang, V., Huang, Y.-I., Achkar, J., Piester, D., Lin, S.-Y., et al. (2018). Use of software-defined radio receivers in two-way satellite time and frequency transfers for UTC computation. Metrologia, 55(5), 685-698.

Kirchner, D. (1991). Two-way time transfer via communication satellites. Proceedings of IEEE, 19, 983-990.

Kouba, J., \& Heroux, P. (2001). Precise point positioning using IGS orbits and clock products. GPS Solutions, 4, 31.

Liang, K., Arias, F., Petit, G., Jiang, Z., Tisserand, L., Wang, Y., et al. (2018). Evaluation of BeiDou time transfer over multiple inter-continental baselines towards UTC contribution. Metrologia, 55(4), 513-525.

Lisdat, Ch., et al. (2016). A clock network for geodesy and fundamental science. Nature Communications, 7, 12443.

McCarthy, D. (2011). Evolution of timescales from astronomy to physical metrology, Metrologia, 48, S132 and all the other papers of this special issue.

Panfilo, G., \& Arias, F. (2019). The Coordinated Universal Time (UTC). Metrologia, 56(4), 042001

Parkinson, B. W., \& Spilker, J. J., Jr. (1996). Global positioning system: Theory and applications. Washington: American Institute of Aeronautics and Astronautics.

Petit, G., Arias, F., Harmegnies, A., Panfilo, G., \& Tisserand, L. (2014). UTCr: A rapid realization of UTC. Metrologia, 51, 33-39.

Petit, G., \& Harmegnies, A. (2019). Tests of Galileo and BeiDou links for UTC. In Proceedings of IFCS-EFTF 2019, Orlando, USA.

Petit, G., \& Jiang, Z. (2008a). GPS Precise point positioning for TAl computation. IJNO. https://doi.org/10.1155/2008/562878.

Petit, G., \& Jiang, Z. (2008b). GPS all in view time transfer for TAl computation. Metrologia, 45, 35-45.

Petit, G., Kanj, A., et al. (2015). 1 × 10 $0^{-16}$ frequency transfer by GPS PPP with integer ambiguity resolution. Metrologia, 52-2, 301.

Ray, J., \& Senior, K. (2003). IGS/BIPM pilot project: GPS carrier phase for time/ frequency transfer and timescale formation. Metrologia, 40, S270-S288.

Ray, J., \& Senior, K. (2005). Geodetic techniques for time and frequency comparisons using GPS phase and code measurements. Metrologia, 42, 215-232.

Riehle, F. (2017). Optical clock networks. Nature Photonics, 11, 25-31. https:// doi.org/10.1038/nphoton.2016.235.

Sesia, I., Signorile, G., Cantoni, E., Cerretto, G., Samperi, A., Tavella, P., et al. (2014) Time metrology in the Galileo Navigation System: The experience of the Italian National Metrology Institute, IEEE International Workshop on Metrology for Aerospace, May 2014, Benevento, Italy (pp. 23-27), https:// doi.org/10.1109/metroaerospace.2014.6865888.

Signorile, G., et al. (2019). GNSS-to-GNSS Time Offsets: A realistic case study, presented at the ION PTTI Precise Time and Time Interval meeting, Reston, VI, Jan 2019.

Tavella, P. (2017). Precise time scales and navigation systems. In M. Milton, C. Williams, P. Tavella, \& N. Deleo (Eds.), Proceedings of the international school of physics "Enrico Fermi" Course 196 - Metrology: From physics fundamentals to quality of life. Amsterdam: IOS Press.

Verhasselt, K., \& Defraigne, P. (2019). Multi-GNSS time transfer based on the CGGTTS. Metrologia, 56, 065003.

\section{Publisher's Note}

Springer Nature remains neutral with regard to jurisdictional claims in published maps and institutional affiliations. 